\title{
Cray Benchmark - calibrating different eDNA methods for crayfish and crayfish plague
}

\author{
Patrik Bohman $\ddagger$ \\ ‡ Swedish University of Agricultural Sciences, UPPSALA, Sweden
}

Corresponding author: Patrik Bohman (patrik.bohman@slu.se)

Received: 02 Mar 2021 | Published: 03 Mar 2021

Citation: Bohman P (2021) Cray Benchmark - calibrating different eDNA methods for crayfish and crayfish plague. ARPHA Conference Abstracts 4: e65359. https://doi.org/10.3897/aca.4.e65359

\begin{abstract}
Many countries are on the verge to integrate eDNA analyses into environmental monitoring. There are no standards yet for this kind of monitoring, and many different methods are used. Still, various methods and protocols have previously proven to be very precise and credible. If we accept that the way to monitor species' eDNA will vary, different methods and actors can be used. To be able to rely on different types of protocols, benchmarking is much needed. We have initiated a project to perform benchmarking on crayfish and crayfish plague in 2021. Previous year a meeting was held in Stockholm where different methods were discussed with researchers, managers and stakeholders from 15 different organisations in 8 countries. The meeting pointed out the necessity for benchmarking to successfully monitor eDNA from crayfish and crayfish plague.
\end{abstract}

\section{Keywords}

Environmental DNA, Benchmark, Monitoring, signal crayfish, noble crayfish, crayfish plague

\section{Presenting author}

Patrik Bohman 


\section{Presented at}

1st DNAQUA International Conference (March 9-11, 2021) 